\title{
Magnetohydrodynamic Free Convection Flow with Thermal Radiation and Chemical Reaction Effects in the Presence of Variable Suction
}

\author{
Halima Usman, Ime Jimmy Uwanta and Sama'ila Kamba Ahmad \\ Department of Mathematics, Usmanu Danfodiyo University, P.M.B. 2346 Sokoto, Nigeria
}

\begin{abstract}
The aim of the present study is to investigate the effect of flow parameters on the free convection and mass transfer of an unsteady magnetohydrodynamic flow of an electrically conducting, viscous and incompressible fluid past an infinite vertical porous plate in the presence of variable suction. The thermal radiation and chemical reaction effects are assumed to exist within the channel. Non dimensional partial differential equations of governing equations of flow are solved numerically using Crank Nicolson finite difference method. The skin friction, heat and mass transfer rates as well as the effects of various parameters on velocity, temperature and concentration profiles are analyzed. The signifiant results from this study are that an increase in the values of radiation parameter and chemical reaction parameter causes a reduction in the velocity, temperature and concentration.
\end{abstract}

\section{Introduction}

The range of free convective flow that occur in nature and in engineering practice is very large and has been extensively considered by many researchers due to its applications in chemically processed industries such as, drying, evaporation at the surface of a water body and food processing. Convection in porous media has applications in geothermal energy recovery, oil extraction, thermal energy storage and flow through filtering devices. Radiative convective flows are encountered in countless industrial and environment processes such as heating and cooling chambers and astrophysical flows. Acharya et al. [1] analyzed free convection and mass transfer flow through a porous medium bounded by vertical infinite surface with constant suction and heat flux. Chamkha [2] studied unsteady MHD convective heat and mass transfer past a semi-infinite vertical moving plate with heat absorption. Cosiderable research work has been published on the study of radiative convective flows in a variety of geometrical configurations with numerical and mathematical models. Chandrakala and Antony [3] discussed radiation effects on MHD flow past an impulsively started infinite vertical plate with variable temperature. Duwairi and Damesh [4] presented a study on heat transfer from radiative vertical porous surfaces. Unsteady free convection flow past a vertical porous plate was investigated by Helmy [5]. Hossain and Takhar [6] reported radiation effect on mixed convection along a vertical plate with uniform surface temperature.

In recent years, progress has been considerably made in the study of heat and mass transfer with chemical reaction due to its applications in many branches of science and engineering. Kandasamy et al. [7] analyzed simultaneously the effects of chemical reaction, heat and mass transfer along a wedge with heat source and concentration in the presence of suction or injection. Kawala and Odda [8] obtained numerical results for the effect of chemical reaction on unsteady natural convection boundary layer flow over a semi-infinite vertical cylinder. Recently, a detailed numerical study was reported by Kishore et al. [9] on the effects of chemical reaction, radiation and magnetic field on the unsteady free convection flow, heat and mass transfer characteristics in a viscous, incompressible and electrically conducting fluid past an exponentially accelerated vertical plate. Mahdy [10] performed an analysis to investigate the effects of chemical reaction and heat generation or absorption on double diffusive convection from vertical truncated cone in a porous media with variable viscosity. Mohammed et al. [11] investigated the effect of similarity solution for MHD flow through vertical porous plate with suction. Patil and Chamkha [12] discussed heat and mass transfer from mixed convection flow of polar fluid along a plate in porous media with chemical reaction. In another article, Rajeshwari et al. [13] presented the effect of chemical reaction on the forced and free convection boundary layer that flows in a semi-infinite expanse of an electrically conducting viscous incompressible fluid past a vertical porous plate.

Rajput and Kumar [14] presented the effect of radiation on MHD flow past an impulsively started vertical plate with variable heat and mass transfer. Raptis and Perdikis [15] described radiation and free convection flow past a moving plate. Saleh et al. [16] examined convective heat and mass transfer characteristics of an incompressible MHD visco-elastic fluid flow immersed 
in a porous medium over a stretching sheet with chemical reaction and thermal stratification. Sarada and Shankar [17] presented a study of chemical reaction effect on an unsteady MHD convective flow past an infinite vertical moving plate embedded in a porous medium with heat source.

Sattar [18] took an account on the effect of free and forced convection boundary layer flow through a porous medium with large suction. Soundalgekar and Takher [19] discussed the effect of radiation on the natural convection flow of a gas past a semi-infinite plate using the CoghyVincentine-Gilles equilibrium model. Rahman [20] discussed the combined effects of internal heat generation and higher order chemical reaction on the non-Darcian forced convective flow of a viscous incompressible fluid with variable viscosity and thermal conductivity over a stretching surface embedded in a porous medium.

The objective of this paper is to study the effects of thermal radiation and chemical reaction on an unsteady MHD free convection heat and mass transfer flow past an infinite vertical plate in presence of variable suction. In this study, the effects of different flow parameters encountered in the equations are also studied. The problem is solved numerically using the implicit finite difference method of Crank Nicolson type, which is more economical from the computational point of view.

\section{Mathematical analysis}

An unsteady MHD free convection heat and mass transfer flow of an electrically conducting, viscous incompressible and radiating fluid over a porous vertical infinite plate with chemical reaction and thermal conductivity is considered. In the Cartesian coordinate, the $x^{\prime}$ - axis is assumed to be along the infinite plate in the direction of the flow and the $y^{\prime}$ - axis is taken normal to it. A magnetic field $B_{0}$ of uniform strength is applied transversely to the direction of the flow as shown in Fig. 1. Initially, the plate and the fluid are at the same temperature $T_{\infty}$ in a stationary condition with concentration level $C_{\infty}$ at all points. For $t^{\prime}>0$, the temperature and concentration at the plate $y^{\prime}=0$ is raised to $T_{w}^{\prime}$ and $C_{w}^{\prime}$ respectively and that of $y^{\prime} \rightarrow \infty$ is lowered to $T_{\infty}^{\prime}$ and $C_{\infty}^{\prime}$ such that $T_{w}^{\prime}>T_{\infty}^{\prime}$ and $C_{w}^{\prime}>C_{\infty}^{\prime}$. It is assumed that the induced magnetic field is negligible in comparison with the applied one which corresponds to a very small magnetic Reynolds number. The flow has significant thermal radiation, chemical reaction and suction effects.

Within the above framework, the governing boundary layer equations of continuity, momentum, energy and concentration under Boussinesq's approximation are as follows:

$$
\frac{\partial v^{\prime}}{\partial y^{\prime}}=0
$$

$$
\begin{gathered}
\frac{\partial u^{\prime}}{\partial t^{\prime}}-v_{0} \frac{\partial u^{\prime}}{\partial y^{\prime}}=v \frac{\partial^{2} u^{\prime}}{\partial y^{\prime 2}}-\left(\frac{\sigma B_{0}^{2}}{\rho}+\frac{v}{k^{*}}\right) u^{\prime} \\
+g \beta\left(T^{\prime}-T_{\infty}^{\prime}\right)+g \beta^{*}\left(C^{\prime}-C_{\infty}^{\prime}\right) \\
\frac{\partial T^{\prime}}{\partial t^{\prime}}-v_{0} \frac{\partial T^{\prime}}{\partial y^{\prime}}=\frac{k_{0}}{\rho C_{p}} \frac{\partial}{\partial y^{\prime}}\left[1+\alpha\left(T^{\prime}-T_{\infty}^{\prime}\right) \frac{\partial T^{\prime}}{\partial y^{\prime}}\right] \\
-\frac{1}{\rho C_{p}} \frac{\partial q_{r}}{\partial y^{\prime}} \\
\frac{\partial C^{\prime}}{\partial t^{\prime}}-v_{0} \frac{\partial C^{\prime}}{\partial y^{\prime}}=D \frac{\partial^{2} C^{\prime}}{\partial y^{\prime 2}}-R^{*}\left(C^{\prime}-C_{\infty}^{\prime}\right)^{n}
\end{gathered}
$$

The initial and boundary conditions are

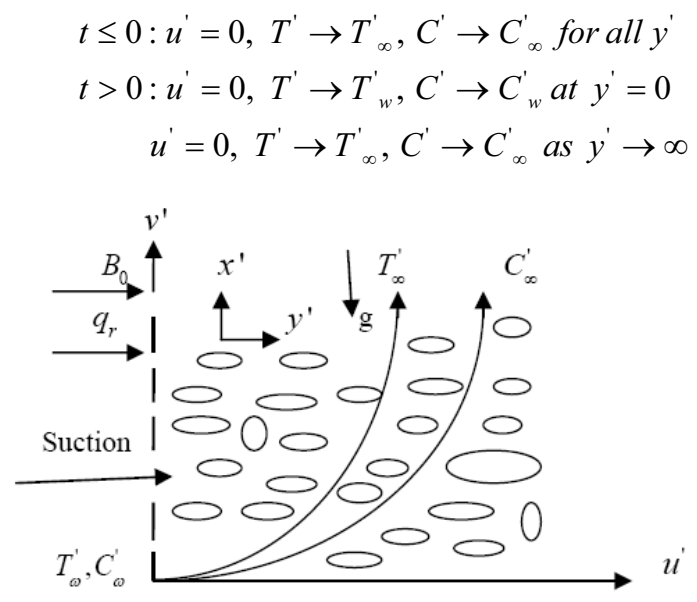

Figure 1. Physical configuration of the problem.

From the continuity equation, it can be seen that $v^{\prime}$ is either a constant or a function of time. Hence, on integrating equation (1), the suction velocity normal to the plate is assumed in the form:

$v^{\prime}=-v_{0}$, where $v_{0}$ is the mean suction velocity which is non-zero positive constant. The negative sign indicates that the suction velocity is directed towards the plate, $v$ is the kinematic viscosity, $g$ is the gravitational force, $\beta$ is the coefficient of thermal expansion, $\rho$ the density of the fluid, $\sigma$ is the electrical conductivity of the fluid, $\beta^{*}$ is the coefficient of concentration expansion, $C_{p}$ is the specific heat capacity at constant pressure, $k^{*}$ is the permeability of the porous medium, $k_{0}$ is the thermal conductivity of the fluid, $\alpha$ is a constant depending on the nature of the fluid, $R^{*}$ is the dimensionless chemical reaction, $n$ is the order of chemical reaction, $D$ is the coefficient of mass diffusivity, $q_{r}$ is the radiative heat flux in the $y$ - direction, $B_{0}$ is the electromagnetic induction.

Using the following non-dimensional quantities: 


$$
\begin{aligned}
& u=\frac{u^{\prime}}{u_{0}}, t=\frac{t^{\prime} u_{0}}{v}, y=\frac{u_{0} y^{\prime}}{v}, \theta=\frac{T^{\prime}-T_{\infty}^{\prime}}{T^{\prime}{ }_{w}-T_{\infty}^{\prime}}, \\
& C=\frac{C^{\prime}-C_{\infty}^{\prime}}{C^{\prime \prime}{ }_{w}-C_{\infty}^{\prime \prime}}, \operatorname{Pr}=\frac{v \rho C_{p}}{k_{0}}, S c=\frac{v}{D}, k=\frac{k^{*} u_{0}{ }^{2}}{v^{2}}, \\
& M=\frac{\sigma B_{0}{ }^{2} v}{\rho u^{2}{ }_{0}}, G r=\frac{v g \beta\left(T^{\prime}{ }_{w}-T_{\infty}^{\prime}\right)}{u^{3}{ }_{0}}, \\
& G c=\frac{\nu g \beta^{*}\left(C^{\prime}{ }_{w}-C_{\infty}^{\prime}\right)}{u^{3}{ }_{0}}, \lambda=\alpha\left(T^{\prime}-T_{\infty}^{\prime}\right), \gamma=\frac{v_{0}}{u_{0}}, \\
& K_{r}=\frac{R^{*}{ }^{2}}{u^{2}{ }_{0}}, R=\frac{16 a \sigma_{0}^{\prime}{ }_{0} v T_{\infty}{ }^{3}}{k u^{2}{ }_{0}} \text {. }
\end{aligned}
$$
form:

Using (6), the equations (1) - (4) take the following

$$
\begin{gathered}
\frac{\partial u}{\partial t}-\gamma \frac{\partial u}{\partial y}=\frac{\partial^{2} u}{\partial y^{2}}-\left(M+\frac{1}{k}\right) u \\
+G r \theta+G c C \\
\frac{\partial \theta}{\partial t}-\gamma \frac{\partial \theta}{\partial y}=\frac{\lambda}{\operatorname{Pr}\left(\frac{\partial \theta}{\partial y}\right)^{2}} \\
+\frac{1}{\operatorname{Pr}}(1+\lambda \theta) \frac{\partial^{2} \theta}{\partial y^{2}}-\frac{R}{\operatorname{Pr}} \theta \\
\frac{\partial C}{\partial t}-\gamma \frac{\partial C}{\partial y}=\frac{1}{S c} \frac{\partial^{2} C}{\partial y^{2}}-K_{r} C^{n}
\end{gathered}
$$

The initial and boundary conditions in dimensionless form are:

$$
\begin{array}{r}
t \leq 0: u=0, \theta=0, C=0, \text { for all } y \\
t>0: u=0, \theta=1, C=1, \text { at } y=0 \\
u=0, \theta=0, C=0 \text { as } y \rightarrow \infty
\end{array}
$$

where $\operatorname{Pr}, S c, M, G r, G c, \lambda, \gamma, R$ and $K_{r}$ are Prandtl number, Schmidt number, Magnetic field parameter, thermal Grashof number, mass Grashof number, variable thermal conductivity, variable suction parameter, radiation parameter and chemical reaction parameter respectively. Of practical interest in many applications of the problem under consideration are the skin friction coefficient, the heat transfer coefficient and the mass transfer coefficient, which are given as

$$
C_{f}=\left(\frac{\partial u}{\partial y}\right)_{y=0}, \quad N u=-\left(\frac{\partial \theta}{\partial y}\right)_{y=0}, S h=\left(\frac{\partial C}{\partial y}\right)_{y=0}
$$

\section{Numerical solutions}

The system of transformed coupled non-linear partial differential equations (7), (8) and (9) together with the initial and boundary conditions (10) have been solved numerically using a finite difference technique of implicit type namely Crank-Nicolson implicit finite difference method. The equivalent finite difference approximations corresponding to equations (7)-(9) are given as follows:

$$
\begin{aligned}
& \left(\frac{u_{i, j+1}-u_{i, j}}{\Delta t}\right)-\frac{\gamma}{2 \Delta y}\left(u_{i+1, j}-u_{i-1, j}\right)= \\
& \frac{1}{2(\Delta y)^{2}}\left(u_{i+1, j+1}-2 u_{i, j+1}+u_{i-1, j+1}+u_{i+1, j}-2 u_{i, j}+u_{i-1, j}\right) \\
& -\left(M+\frac{1}{k}\right) u_{i, j}+G r\left(\theta_{i, j}\right)+G c\left(C_{i, j}\right) \\
& \left(\frac{\theta_{i, j+1}-\theta_{i, j}}{\Delta t}\right)-\frac{\gamma}{2 \Delta y}\left(\theta_{i+1, j}-\theta_{i-1, j}\right)= \\
& \frac{H}{2 \operatorname{Pr}(\Delta y)^{2}}\left(\theta_{i+1, j+1}-2 \theta_{i, j+1}+\theta_{i-1, j+1}+\theta_{i+1, j}-2 \theta_{i, j}+\theta_{i-1, j}\right) \\
& +\frac{\lambda}{\operatorname{Pr}(\Delta y)^{2}}\left(\theta_{i+1, j}-\theta_{i, j}\right)^{2}-\frac{R}{\operatorname{Pr}}\left(\theta_{i, j}\right) \\
& \left(\frac{C_{i, j+1}-C_{i, j}}{\Delta t}\right)-\frac{\gamma}{2 \Delta y}\left(C_{i+1, j}-C_{i-1, j}\right)= \\
& \frac{1}{2 S c(\Delta y)^{2}}\left(C_{i+1, j+1}-2 C_{i, j+1}+C_{i-1, j+1}+C_{i+1, j}-2 C_{i, j}+C_{i-1, j}\right) \\
& -K_{r}\left(C_{i, j}\right)^{n}
\end{aligned}
$$

the initial and boundary conditions are :

$$
\begin{aligned}
& u_{i, j}=0, \theta_{i, j}=0, C_{i, j}=0 \text { for all } j=0 \\
& u_{0, j}=0, \theta_{0, j}=1, C_{0, j}=1 \\
& u_{h, j}=0, \theta_{h, j}=0, C_{h, j}=0
\end{aligned}
$$

Equations (11), (12) and (13) are simplified as follows

$$
\begin{gathered}
-U_{1} u_{i-1, j+1}+U_{2} u_{i, j+1}-U_{1} u_{i+1, j+1}=U_{3} u_{i-1, j} \\
+U_{4} u_{i, j}+U_{5} u_{i+1, j}+U_{6} \theta_{i, j}+U_{7} C_{i, j} \\
-T_{1} \theta_{i-1, j+1}+T_{2} \theta_{i, j+1}-T_{1} \theta_{i+1, j+1}=T_{3} \theta_{i-1, j} \\
+\left(T_{4}-T_{7}\right) \theta_{i, j}+T_{5} \theta_{i+1, j}+T_{6}\left(\theta_{i+1, j}-\theta_{i, j}\right)^{2} \\
-V_{1} C_{i-1, j+1}+V_{2} C_{i, j+1}-V_{1} C_{i+1, j+1}=V_{3} C_{i-1, j} \\
+V_{4} C_{i, j}+V_{5} C_{i+1, j}-V_{6}\left(C_{i, j}\right)^{n}
\end{gathered}
$$

where

$$
\begin{aligned}
& r_{1}=\frac{\gamma \Delta t}{\Delta y}, r_{2}=\frac{\Delta t}{(\Delta y)^{2}}, r_{3}=2 \Delta t, r_{4}=M+\frac{1}{k}, H=1+\lambda \theta, U_{1}=r_{2}, \\
& U_{2}=2\left(1+r_{2}\right), U_{3}=r_{2}-r_{1}, U_{4}=2-2 r_{2}-r_{3} r_{4}, U_{5}=r_{1}+r_{2}, \\
& U_{6}=r_{3} G r, U_{7}=r_{3} G c, V_{1}=r_{2}, V_{2}=2\left(S c+r_{2}\right), V_{3}=r_{2}-r_{1} S c, \\
& V_{4}=2\left(S c-r_{2}\right), V_{5}=r_{1} S c+r_{2}, V_{6}=r_{3} S c K r, T_{1}=H r_{2}, \\
& T_{2}=2\left(\operatorname{Pr}+H r_{2}\right), T_{3}=H r_{2}-r_{1} \operatorname{Pr}, T_{4}=2\left(\operatorname{Pr}-H r_{2}\right), T_{5}=r_{1} \operatorname{Pr}+H r_{2}, \\
& T_{6}=2 \lambda r_{2}, T_{7}=r_{3} R .
\end{aligned}
$$




\section{Results and discussion}

In order to get a physical view of the present problem, numerical results for velocity profiles $(u)$, temperature profiles $(T)$, concentration profiles $(C)$, skin-friction $\left(C_{f}\right)$, rate of heat transfer in terms of Nusselt number $(\mathrm{Nu})$ and rate of mass transfer in terms of Sherwood number (Sh) have been computed with help of graphs by assigning numerical values for different values of pertinent parameters. The following values are used for the investigation: $G r=G c=2.0, M=K_{r}=k=t=0.1$,

$$
\operatorname{Pr}=0.71, S c=0.6, R=3.0, n=5.0 \text { and } \lambda=\gamma=0.5
$$

Fig. 2 and 3 depicts the fluid velocity distribution profiles with variation of porosity parameter $(k)$ and magnetic parameter $(M)$. On increasing $k$, it is analyzed that the fluid velocity distribution in this figure increases. It can be seen in Fig. 3 that increasing the magnetic parameter $(M)$ causes a fall in the velocity of the fluid. The effect of transverse magnetic field on an electrically conducting fluid slows down the motion of the fluid. Fig. $4,5,6,7$ and 8 plot the variation of the fluid velocity, temperature and concentration distributions for various values of suction parameter $(\gamma)$ and chemical reaction parameter $\left(K_{r}\right)$. It is observed that as $\gamma$ and $K$ increases, both fluid velocity, temperature distribution and concentration distribution decreases respectively. Fig. 9, 10 and 11 presented the growth of the fluid boundary layer and the thermal distribution profiles with the variation of radiation parameter $(R)$ and Prandtl number (Pr) respectively. It is observed that increasing either $R$ or Pr produces a significant decrease in the velocity and thermal condition of the fluid. Fig. 12 shows that increasing Schmidt number $(S c)$ tends to decrease the fluid concentration boundary layer thickness. Fig. 13, 14 and 15 represent the variation of skin friction against magnetic parameter $(M)$, the Nusselt number against radiation parameter $(R)$ and the Sherwood number against chemical reaction parameter $\left(K_{r}\right)$ for various values of Prandtl number ( $\mathrm{Pr}$ ). It is evident from Fig. 13 that higher values of $M$ and Pr reduce the skin friction while the Nusselt number tend to decrease rapidly as the Prandtl number is increased and the Sherwood number increases with increasing Prandtl number.

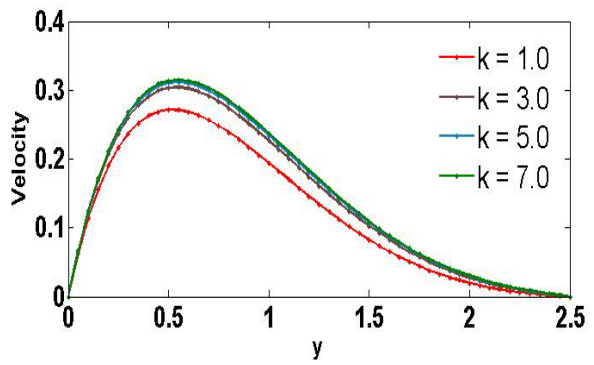

Figure 2. Variation of velocity with $(k)$.

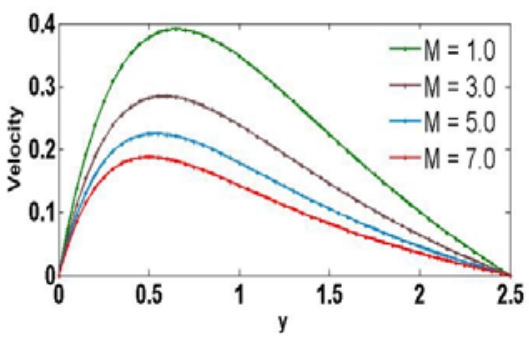

Figure 3. Variation of velocity with ( $M)$.

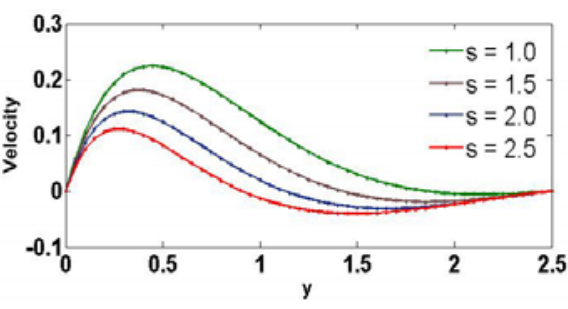

Figure 4. Variation of velocity with $(\gamma=s)$.

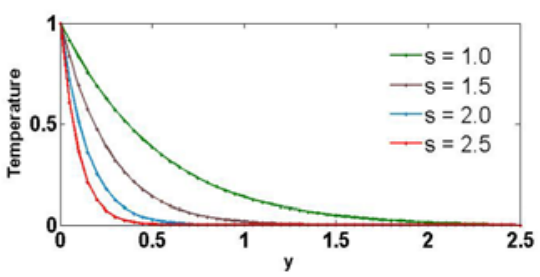

Figure 5. Variation of temperature with $(\gamma=s)$

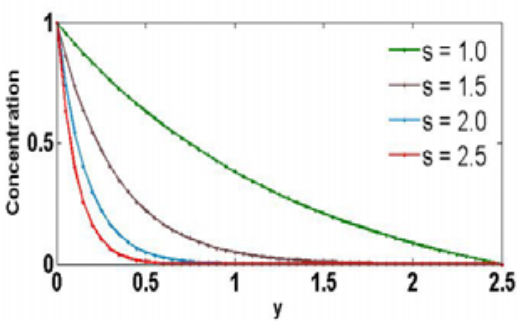

Figure 6. Variation of concentration with $(\gamma=s)$.

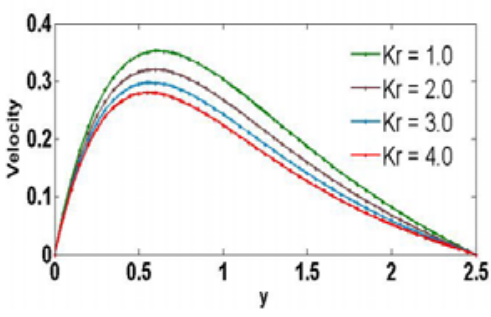

Figure 7. Variation of velocity with $\left(K_{r}\right)$.

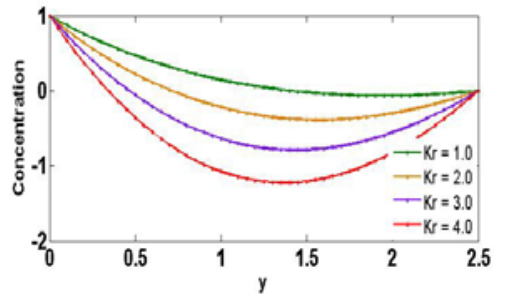

Figure 8. Variation of concentration with $\left(K_{r}\right)$. 


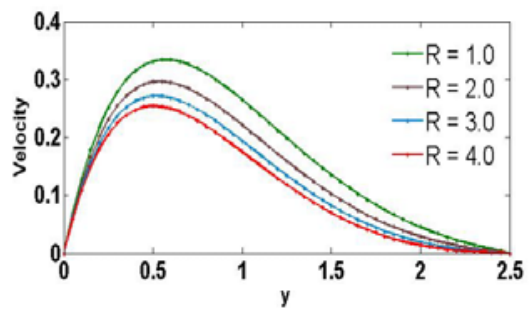

Figure 9. Variation of velocity with $(R)$.

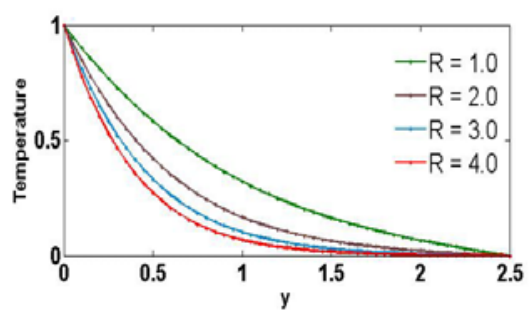

Figure 10. Variation of temperature with $(R)$.

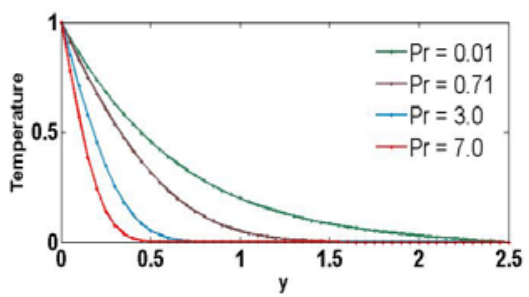

Figure 11. Variation of temperature with $(\mathrm{Pr})$.

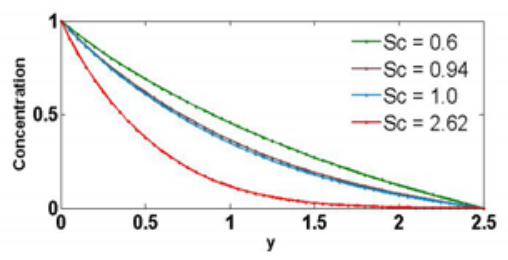

Figure 12. Variation of concentration with $(S c)$.

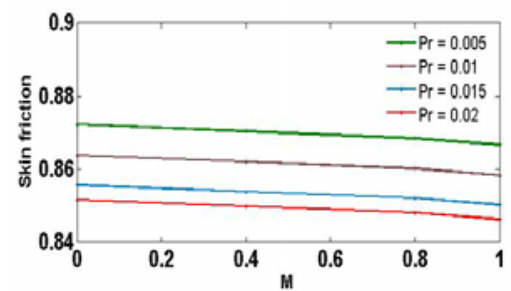

Figure 13. Variation of skin friction with ( $M \& \operatorname{Pr}$ ).

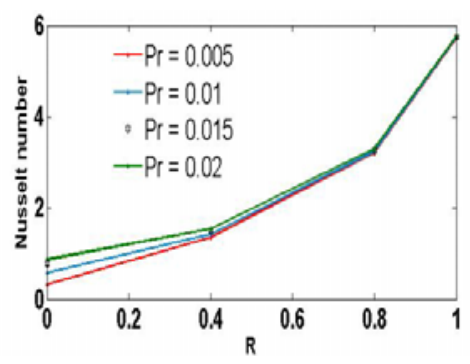

Figure 14. Variation of Nusselt number with $(R \& \operatorname{Pr})$.

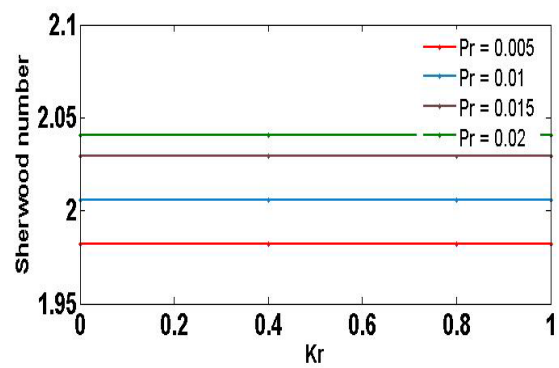

Figure 15. Variation of Sherwood number with $\left(K_{r} \& \operatorname{Pr}\right)$.

\section{Conclusions}

This paper investigates the effects of radiation and chemical reaction on unsteady MHD free convection flow past an infinite vertical plate with variable suction. The governing partial differential equations are solved by an implicit finite difference method of Crank Nicolson type. The conclusions from the present study are as below: Velocity profile increases with increasing $\gamma$. It is also interesting to note that increase in $M, R, K$ and $\gamma$ decelerates the flow velocity. An increasing $\operatorname{Pr}, R, K$, and $\gamma$ decrease the temperature profile of the fluid flow field at all points. Concentration of the fluid decreases with the increasing values of $\gamma, S c$ and $K_{r}$.Skin friction and Nusselt number tend to decrease rapidly as the $\operatorname{Pr}$ is increased, while the Sherwood number increases with increasing Pr.

This type of flow can be investigated in different geometries, like cylindrical coordinates, Couette flow etc. and effects of Navier slip on the flow system.

$C$ - Concentration

$C_{p}$ - Specific heat at constant pressure

$D$ - Mass diffusivity

$g-$ Acceleration due to gravity

$G r$ - Grashof number

$G c$ - Solutal Grashof number

$k$ - Porous parameter

$\mathrm{Nu}$ - Nusselt number

Pr - Prandtl number

Sc - Schmidt number

$R$ - Radiation parameter

$K_{r}$ - Chemical reaction parameter

$n$ - Order of chemical reaction

$T$-Temperature

$C_{f}$-Skin friction

$S h$ - Sherwood number

$u, v$-velocities in the $x$ and $y$-direction respectively

$x, y$-Cartesian coordinates along the plate and normal to it respectively

$B_{0}$ - Magnetic field of constant strength

$M$ - Magnetic field parameter

\section{Greek Letters}


$\beta$ - Coefficient of expansion with concentration

$\beta$ - Coefficient of thermal expansion

$\sigma_{0}^{\prime}$ - Stefan-Boltzmann constant

$\sigma$ - Scalar electrical conductivity

$\rho$ - Density of fluid

$\theta$ - Dimensionless temperature

$v$ - Kinematic viscosity

$\gamma$ - Suction parameter

$\lambda$ - Variable thermal conductivity

\section{Subscript}

$\infty$ - condition at infinity

\section{References}

1. M. Acharya, G. C. Dash, L. P. Singh. Magnetic field effects on the free convection and mass transfer flow through porous medium with constant suction and constant heat flux, Int. J. of Pur. \& Appl. Maths., 31, 1 1-18 (2000)

2. A. J. Chamkha. Unsteady MHD convective heat and mass transfer past a semi-infinite vertical permeable moving plate with heat absorption, Int. J. of Eng. Sci., 42, 217-230 (2004)

3. P. Chandrakala, S. R. Antony. Radiation effects on MHD flow past an impulsively started infinite vertical plate with variable temperature, Int. J. of Maths, 48, 2 167-175 (2006)

4. H. M. Duwairi, R. A. Damesh. MHD natural convection heat transfer from radiative vertical porous surfaces, Heat Mass Trans., 31, 243-248 (1996)

5. K. A. Helmy. MHD unsteady free convection flow past a vertical porous plate, ZAMM, 78, 4, 225-270 (1998)

6. M. A. Hossain, H. S. Takhar. Radiation effect on mixed convection along a vertical plate with uniform surface temperature, Heat Mass Trans., 31, 243-248 (1996)

7. R. Kandasamy, K. Perisamy, P. K. Sivagnana. Effects of chemical reaction, heat and mass transfer along a wedge with heat source and concentration in the presence of suction or injection, Int. J. of Heat and Mass Trans., 48, 1388-1394 (2005)

8. A. M. Kawala, S. N. Odda. Numerical investigation of unsteady free convection on a vertical cylinder with variable heat and mass flux in the presence of chemical reactive species, Adv. Pur. Maths, 3, 1A 183-189 (2013)
9. P. M. Kishore, N. V. Prasada, S. V. Varma, S. Vantarakamana. The effects of radiation and chemical reaction on unsteady MHD free convection flow of viscous fluid past an exponentially accelerated vertical plate, Int. J. Phys. and Math. Sci. 4, 1 300-317 (2013)

10. A. Mahdy. Effect of chemical reaction and heat generation/absorption on double-diffusive convection from a vertical truncated cone in a porous media with variable viscosity, Int. Comm. Heat and Mass Trans., 37, 5 548-554 (2010)

11. F. Mohammad, O. Masatiro, S. Abdus, A. Mohamud. Similarity solution for MHD flow through vertical porous plate with suction, J. Comp. and Appl. Mech. 6, 1 15-25 (2005)

12. P. M. Patil, A. J. Chamkha. Heat and mass transfer from mixed convection flow of polar fluid along a plate in porous media with chemical reaction, Int. J. Num. Meth. Heat Fluid Flow, 23, 5, 899-926 (2013)

13. R. Rajeshwari, B. Jothiram, V. K. Nelson. Chemical reaction, heat and mass transfer on non linear MHD boundary layer flow through a vertical porous plate in the presence of suction, Appl. Math. Sci. 3, 50 2469-2480 (2009)

14. U. S. Rajput, S. Kumar. Radiation effects on MHD flow past an impulsively started vertical plate with variable heat and mass transfer, J. Appl. Mathe. Mech. 8, 66-85 (2012)

15. A. Raptis, C. Perdikis. Radiation and free convection flow past a moving plate, Appl. Mech. Eng. 44, 817821 (1999)

16. M. A. Saleh, A. A. Mohamed, S. E. Mahmoud. Heat and mass transfer in MHD visco-elastic fluid flow through porous medium over a stretching sheet with chemical reaction, J. Appl. Maths. 1, 446-455 (2010)

17. Sarada, Shankar. Chemical reaction effect on an unsteady MHD convective flow past an infinite vertical moving plate embedded in a porous medium with heat source, J. Glo. Res. Math. Arch. 1, 5 63-81 (2013)

18. M. A. Sattar. Free and forced convection boundary layer flow through a porous medium with large suction, Int. J. En. Res. 17, 1-7 (1993)

19. V. M. Soundalgekar, H. S. Takher. Radioactive convective flow past a semi-infinite vertical plate, Mod. Meas. Cont. 51, 31-40 (1992)

20. M. M. Rahman. Combined effects of internal heat generation and higher order chemical reaction on the non-Darcian forced convective flow of a viscous incompressible fluid with variable viscosity and thermal conductivity over a stretching surface embedded in a porous medium, Can. J. Chem. Eng. 90, 1631-1644 (2012) 